\title{
Electronic Health Records: a Survey of the Experiences and Expectations of Irish Dermatologists
}

\author{
Dmitri Robert Wall $\dagger+$ \\ Department of Dermatology $\dagger$ \\ University Hospital Limerick, Ireland \\ Email: dmitri.wall@gmail.com
}

\author{
Bridget Kane
Kashif Ahmad $\dagger \quad$ Bart Ramsay $\dagger$
Department of Computer Science $\$$
Trinity College Dublin, Ireland

\begin{abstract}
The potential of Electronic Health Records (EHRs) is widely recognised, however studies assessing dermatology endusers expectations and perceived benefits are lacking.

We report on a survey of 142 physicians working in Dermatology in Ireland. Eight question stems assess experience with EHRs and expectations of an ideal system for use in Dermatology. $61(43 \%)$ physicians responded, of which 27 (44 $\%)$ describe their experience with EHRs. 6 (33\%) of 18 suggest components of an ideal dermatology EHR (ease of data inputting, integration with laboratory systems and radiology, integration with administrative systems, photography, drawing tools and standardised nomenclature) rate between very important and vital.

The usefulness of an ideal dermatology EHR rates between very and extremely useful across all categories proposed, with safety, building a National network and demonstrating the work performed by a department rating highest.

This survey highlights the core needs of dermatology end-users, whose integral involvement is essential to ensure successful EHR implementation.
\end{abstract}

\section{INTRODUCTION}

"The perfect Electronic Health Record (EHR) offers the potential of providing an overall picture of a patient's health by linking to hospital records, laboratories, and physicians. Treatments prescribed by other physicians would be available at all times, and redundant laboratory studies and potentially harmful drug interactions could be avoided" [1]. Internationally, EHRs are widely heralded as a tool that can dramatically transform the practice of medicine. Some countries have invested heavily, such as the United States of America where $\$ 19$ billion has been appropriated to stimulate the widespread adoption of EHRs [2]. Ireland's health authority, the Health Service Executive of Ireland (HSE), has acknowledged the EHR as the number one innovative change required by the clinical services [3]. It is vital that EHRs rate highly with respect to usability, as lessons learned from other countries stress the importance of engaging stakeholders to ensure the successful implementation of EHRs [4], [5].

In this context, we undertook a survey of physicians working in Dermatology in Ireland regarding their experience with electronic health records and their perspective on requirements for an ideal EHR to best serve the practice of Dermatology.

\section{Methodology}

This is a non-clinical survey of colleagues to ascertain their experience with electronic health records and their opinions regarding the requirements of an electronic health record to best serve dermatology. A survey using the online tool SurveyMonkey®consisting of 8 questions is used. Questions are based on literature relating to electronic health records in dermatology and the authors' experience of developing a modular electronic patient record. Questions 1 through 3 identify the professional position of the responder, whether they work in the public, private or both healthcare systems, and their experience with EHRs. Further clarification is sought on an individual basis where a response is given in this regard. Question 4 asks respondents to rate the importance of 18 possible components of an ideal dermatology specific EHR on a five-point scale. The five options are 'should not be included', 'not important', 'somewhat important', 'very important' and 'vital'; scoring is 1-5. Respondents were also encouraged to suggest any further components that were not listed. Question 5 asks respondents to rate how useful they feel that a dermatology EHR would be on a scale of 'not useful', 'somewhat useful', 'very useful', 'extremely useful', which are scored 1-4, across 9 categories. (A fifth option was a non-answer.) The 9 categories used are 'demographics', 'research', 'safety', 'work performed', 'building a National network', 'teaching', 'continuous professional development', 'meetings' and 'security'. The relevance of each area was explained and an example given of each. An option was available for respondents to suggest further components, or categories, that were not listed. Question 6 asks respondents to comment on the concept of generating a National web based dermatology EHR. Question 7 offers respondents an option to leave general comments and question 8 allows for respondents to leave contact details.

The survey was piloted locally and then, in May 2012, a web link was distributed to all members of the Irish Association of Dermatologists (IAD) throughout the country, and to trainees known to be working in the specialty of dermatology in Ireland. The Irish Association of Dermatologists is an all- 


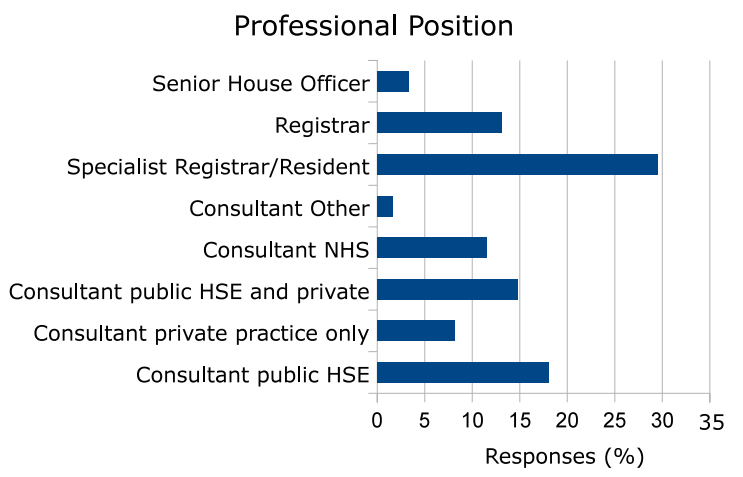

Fig. 1. a) Respondents' Professional Role (left)

Ireland professional body which is reasonably expected to have the majority of dermatologists in Ireland within its membership. Two weeks later, a reminder was sent to all contacted and data collection was completed within one month after commencing the survey.

\section{RESULTS}

Of 142 who received a link, $61(43 \%)$ responded and 56 $(92 \%)$ of those completed the survey in full.

The largest category of respondents are consultants (33; $54 \%)$ and include those in public $(11 ; 18 \%)$, private $(5$; $8.2 \%)$ and mixed practice $(9 ; 14.8 \%)$ as well as 4 in the National Health Service (NHS) (11.5\%), and one in another jurisdiction (1.6\%) (Figure 1). 18 (29.5\%) respondents are Specialist Registrars / Residents.

$27(44 \%)$ respondents describe their experience with EHRs; in $6(10 \%)$ cases, this experience was gained while investigating getting an EHR. $2(3 \%)$ respondents, both in private practice, have paper free practices (Figure 1).

With respect to previous experience with EHRs, 20 responses identified 14 systems. Of these, 8 systems are being utilized within the Republic of Ireland. These include three commercial solutions; two designed for use in primary care and one for use as a generic, but customizable solution for speciality care. Two hospital-based solutions that rely mainly on scanning for clinical documentation are noted, along with a diabetes care management system. Three further systems involve the utilization of software to create letters, which can be accessed as a source of patient information. Problems noted include timeliness of scanning letters, difficulty in implementing the system, duplication of data where systems co-existed with paper notes, difficulty of data entry and interoperability with other systems.

Six systems used internationally are mentioned including both European and US based systems ranging from complete EHRs to systems for creating and accessing documentation in a sequential free-text format. Difficulties noted include being cumbersome, suffering from service interruptions that has lead to frustration and inefficiency, an interface that can disrupts the patient-clinician communication and difficulties locating documents that have been scanned from patients' paper notes.
Experience with EHRs

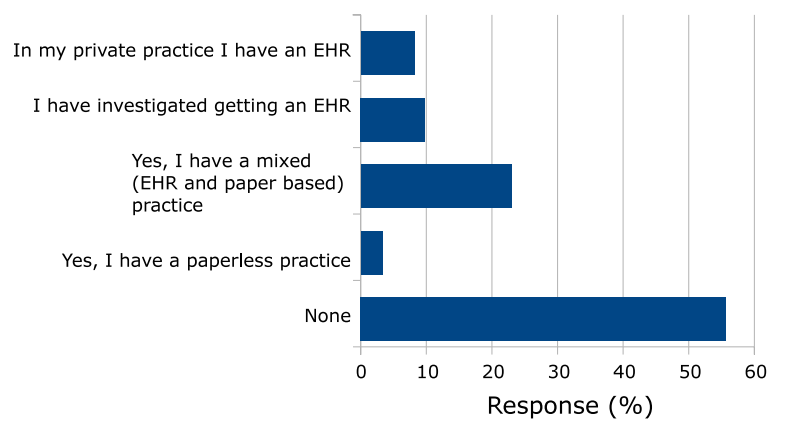

Fig. 1b) Respondents' Experience with Electronic Health Records (right)

58 respondents rate the 18 components identified in question 4 on a scale between 1 and 5 (Figure 2). Of these, 6 (33\%) components rate between very important and vital; in order of average rating, these are 'Ease of data input' (4.47), "Integration with lab systems and radiology" (4.38), "Integration with administrative systems" (4.31), "Photography" (4.28), "Standardised nomenclature" (4.14) and "HIPE (Hospital In Patient Enquiry) coding integration" (4.1) which is the system that is used in Ireland to help allocate hospital funding. Of the remaining $12(67 \%)$ components, all rate between somewhat important and very important. Seven respondents suggest further components such as alert mechanisms, an ability to personalise the interface and a facility to e-mail patients.

57 respondents rate the usefulness of an EHR on a scale between 1 and 4. All areas we propose rate between very and extremely useful (Fig.4). Safety, for example the ability to identify patients on withdrawn drugs or highlighting possible drug interactions, rates highest (3.56). The ability to build a National network and to demonstrate the work performed by a department are the next most highly rated areas (3.54). The potential ability to improve research, including the ability to audit and develop registries scored 3.51 while the potential to improve security, such as by avoiding the need to e-mail confidential information scored 3.46. Improving the ability to collect demographic information, support Continuous Professional Development, meetings and teaching scored 3.44, 3.39, 3.33 and 3.21 respectively.

26 responses are given with respect to the concept of building a National web based EHR. 24 are supportive, expressing beliefs such as that a dermatology specific EHR is extremely advantageous to dermatology Nationally since we are a small country. 2 cautious replies suggest that the process may be complicated, and that it may not be necessary to have a National network.

14 responses $(85 \%)$ provide general feedback within the survey. These include significant support for the idea of a dermatology specific EHR, noting that there could be substantial benefits in efficiency and safety. Respondents identify a long term cost effectiveness, while informing us that similar principles are used to drive research in general practice. Two responses raise concerns as to the ability of different EHRs to 


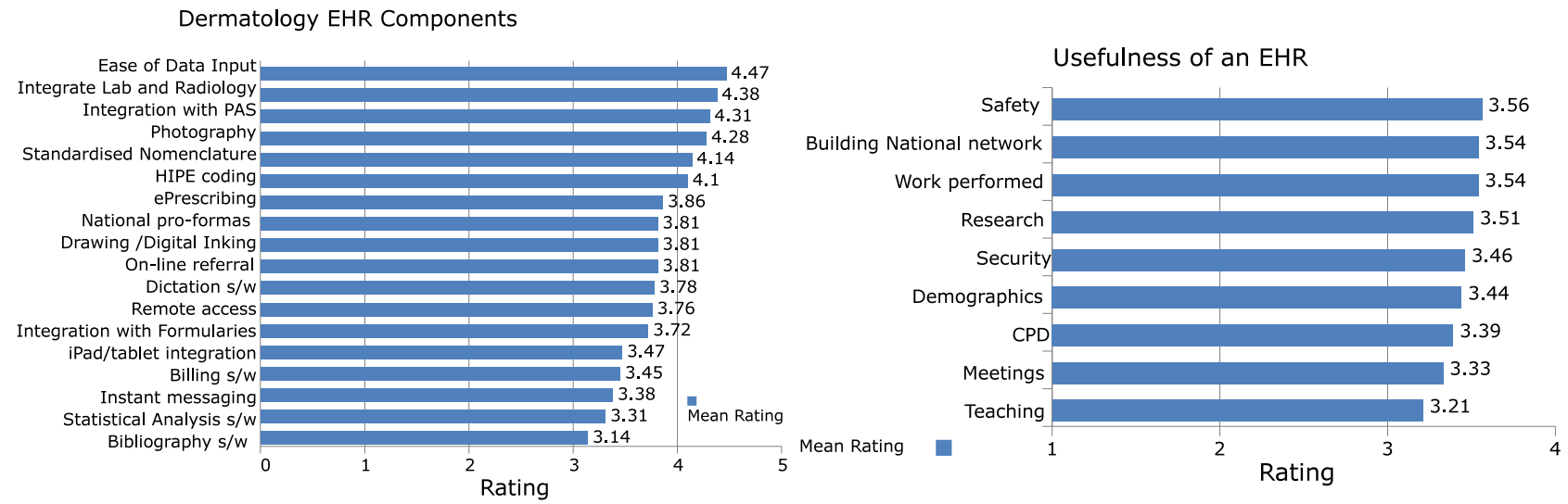

Fig. 2. Identified Requirements of a Dermatology EHR are listed on the left. The Experienced Usefulness of dermatology EHR is represented on the right.

integrate with current systems, in addition to issues of cost, complexity and security. All 24 respondents submitted their contact details.

\section{Discussion}

The response rate of $43 \%$, though it may sound relatively low is a good yield of return for such a survey, and represents a large proportion of the consultant dermatologists practicing in Ireland. This interest in the survey, we feel, demonstrates an interest in an area that is likely to be a contentious, but significant tool in the delivery of dermatological care in the coming years. The percentage of respondents describing no past experience of EHRs is large, but correlates with what is known about EHRs in Ireland; that despite growing international impetus there is still little penetration within the Irish healthcare system. Of the systems described as being in current use in Ireland, many are general practice based systems. This correlates with reports that point to general practice in Ireland implementing "computer systems" ahead of secondary and tertiary care for recording consultations [6].

Two consultants, both based in private practice, have paper free systems, which is interesting in the context of expressed concerns about efficiency and value for money. A number of the other systems noted are systems that are used in other specialties; indeed only one system identified is being used specifically in the area of dermatology; and that system is primarily a collection of patient letters. These results imply that the true experience with genuine EHRs is much lower than the reported $41 \%$. Where genuine experience did occur with true EHRs, it is interesting to note that physicians experience significant difficulties with systems in use internationally, but still recognise the significant benefits that they enable, which is in keeping with international experience [4].

One hospital-based EPR system mentioned is of particular significance: comments noting significant duplication of work highlight difficulties experienced where a system has only partial integration with legacy systems, and does not fully replace paper notes. One respondent described it: "...frustrations working with a half paperless, half electronic system ... ends up with duplication and doubling of the work".

All the components of a dermatology specific EHR we propose are viewed as being either very important or vital components; those most valued relate directly to entry and availability of clinically useful information. The vital importance of usability is raised through several of the questions, and comments reinforce the issue of designing a system fit for use in a busy hospital clinic. Over $80 \%$ of the respondents consider it 'Vital' or 'Very Important' that the system would allow for 'Ease of data entry' and this feature emerges as the dermatologists' highest priority. As one respondent put it "The system is cumbersome, and sometimes the server is down - and if this happens in the middle of a clinic it is very annoying and wastes a lot of time".

As would be expected in a particularly visual specialty such as dermatology, support for photography capture, display and cataloguing within an EHR is considered very important or vital by $84 \%$ of respondents. Related to the image needs of dermatologists is a drawing tool to allow for drawing on a body map, or to draw intra-operative diagrams. One dermatologist said: "[It is] useful for Mohs" and to document [the] reconstruction used". With respect to the usefulness of a dermatology EHR, its greatest value pertains to its potential to improve patient safety and disseminate information. There is also an emphasis placed on its use as a research tool and to demonstrate the work performed by a department.

A striking feature that the information revolution has highlighted is the value of interconnectivity and this is represented by the vast majority of respondents noting the importance of developing a National, networked EHR solution, though concerns relating to security are expressed. Concerns relating to the cost and complexity of developing a Nationally networked EHR are expressed. This resonates with the UK where a National EHR has as yet failed to materialise despite significant investment.

\footnotetext{
${ }^{1}$ Mohs surgery, or chemosurgery, is microscopically controlled surgery used to treat skin cancer
} 


\section{CONCLUSION}

EHRs have long been considered to be capable of revolutionizing the practice of medicine [7]. Despite huge potential, evidence of their success is mixed [8]. End user buy-in has been recognized as a significant aspect in relation to their successful implementation [5]. While informative articles in the literature written by experts have outlined the specific EHR needs in respect to dermatology, there are few studies assessing the opinions of larger groups of dermatologist endusers [1], [9]. We feel that our study provides important information in this regard that is not only relevant within a country that is largely EHR naive such as Ireland, but also to the wider dermatology community with respect to defining the essential components and functionality of a system to suit the distinctive requirements of Dermatology. We believe it is essential that dermatologists are involved in the development of a Dermatology specific EHR to enable it to be a tool that enhances patient care and research, rather than waiting for one designed by non-dermatologists, which will not meet the specialty's unique medical and surgical case mix. Though the focus of this survey is dermatology, many concepts that define the utility of an EHR for one specialty are applicable to other specialties. As such, we feel that the results of this survey are of significance to a wider medical and technical audience. Indeed, we encourage all medical specialties to define their specialist needs to enable the broad representation required to ensure that the true transformative potential of EHRs is met. This is of particular significance in Ireland, where EHR development has been identified as a priority but core components and functionality are still being defined.

\section{ACKNOWLEDGEMENT}

We wish to extend our thanks to those members of the IAD who participated in the survey.

\section{REFERENCES}

[1] J. A. Grosshandler, B. Tulbert, M. D. Kaufmann, A. Bhatia, and R. T. Brodell, "The electronic medical record in dermatology," Archives of Dermatology, vol. 146, no. 9, pp. 1031-1036, 2010, 10.1001/archdermatol.2010.229.

[2] US Congress," "The American Recovery and Reinvestment Act of 2009: Health information technology for economic and clinical health act (title xiii of division a and title iv of division b of the american recovery and reinvestment act of 2009," 2009.

[3] T. Shannon, "National clinical programmes: Aligning process improvements with information technologies. strategic framework proposal," University College London, Report, 2009.

[4] J. Corrao, Natalie, A. G. Robinson, M. A. Swiernik, and A. Naeim, "Importance of testing for usability when selecting and implementing an electronic health or medical record system," Journal of Oncology Practice, vol. 6, no. 3, pp. 120-124, 2010.

[5] S. Silow-Carrol, J. N. Edwards, and D. Rodin, "Using electronic health records to improve quality and efficiency: the experiences of leading hospitals," The Commonwealth Fund, vol. 1608, no. 17, pp. 1-40, 2012.

[6] S. Mc Hugh, J. OKeeffe, A. Fitzpatrick, A. de Sin, M. OMullane, I. Perry, and C. Bradley, "Diabetes care in ireland: A survey of general practitioners," Primary Care Diabetes, vol. 3, no. 4, pp. 225-231, 2009.

[7] Institute of Medicine, Committee on Quality of Health Care in America, "Crossing the quality chasm: A new health system for the 21st centuryis," Institute of Medicine, Report, 2001.
[8] C. M. DesRoches, E. G. Campbell, C. Vogeli, J. Zheng, S. R. Rao, A. E. Shields, K. Donelan, S. Rosenbaum, S. J. Bristol, and A. K. Jha, "Electronic health records' limited successes suggest more targeted uses," Health Affairs, vol. 29, no. 4, pp. 639-646, 2010.

[9] M. D. Kaufmann and S. Desai, "Special requirements for electronic health records in dermatology," Seminars in Cutaneous Medicine and Surgery, vol. 31, pp. 160-2, 2012. 